\title{
Otimização do processamento para a fabricação de filtros no sistema $\mathrm{Al}_{2} \mathrm{O}_{3}-\mathrm{SiC}$
}

\section{(Processing optimization for production of $\mathrm{Al}_{2} \mathrm{O}_{3}$-SiC ceramic filters)}

\author{
V. R. Salvini, A. M. Pupim, M. D. M. Innocentini, V. C. Pandolfelli \\ Departamento de Engenharia de Materiais - DEMa \\ Universidade Federal de S. Carlos - UFSCar \\ Rod. Washington Luiz, km 235, S. Carlos, SP, 13565-905 \\ pvrs@iris.ufscar.brouvicpando@power.ufscar.br
}

\begin{abstract}
Resumo
Neste trabalho avaliou-se o efeito das condições de processamento cerâmico na permeabilidade e resistência mecânica de filtros celulares. Esta avaliação compreendeu a fabricação e caracterização de filtros cerâmicos de 8 ppi (poros por polegada linear) no sistema $\mathrm{Al}_{2} \mathrm{O}_{3}-\mathrm{SiC}$ produzidos a partir de suspensões cerâmicas com diferentes ajustes granulométricos. $\mathrm{O}$ ajuste granulométrico tem por objetivo otimizar o empacotamento das partículas nos filamentos sólidos e, conseqüentemente, melhorar as propriedades mecânicas do filtro, porém sem comprometer a permeabilidade do mesmo. Neste estudo, a caracterização compreendeu tanto as propriedades reológicas das suspensões selecionadas bem como a caracterização física, fluidodinâmica e mecânica dos filtros obtidos.
\end{abstract}

Palavras-chave: filtros cerâmicos, processamento, permeabilidade, propriedades mecânicas.

\section{INTRODUÇÃO}

É crescente o uso de filtros cerâmicos na indústria metalúrgica para a remoção de impurezas de ligas metálicas fundidas. O material utilizado neste caso deve ser refratário, resistir a tensões termomecânicas, ser eficiente na remoção de impurezas e inerte em relação ao fundido que está sendo filtrado. Além desta aplicação, os filtros cerâmicos também são usados no setor automobilístico para remover partículas de fuligem, especialmente dos motores a diesel. Também neste caso o material deve ser refratário, permeável, resistente ao choque térmico e eficiente quanto à retenção de impurezas [1-3].

Os principais critérios de avaliação de filtros cerâmicos celulares são a permeabilidade, a eficiência na retenção de impurezas e a resistência mecânica. Idealmente, o filtro cerâmico deveria remover o máximo de impurezas com a mínima resistência ao fluido de arraste. Uma maneira de se obter alta permeabilidade seria aumentar o volume de vazios na estrutura (porosidade). Contudo, esta opção compromete tanto a eficiência de coleta de impurezas quanto a resistência mecânica do filtro.

\begin{abstract}
The objective of this work was to evaluate the effect of processing parameters on the permeability and mechanical resistance of cellular ceramic filters. Analyses included

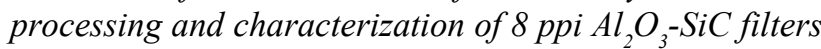
produced from ceramic suspensions with different particle size distributions. The purpose was to optimize the particle packing in the solid struts and thus improve the mechanical properties of the filter without reducing its permeability. Characterization included evaluation of rheological properties of suspensions as well as measurements of porosity, permeability and mechanical resistance of the produced filters.
\end{abstract}

Keywords: ceramic filters, processing, permeability, mechanical properties.

A microestrutura de materiais celulares obtidos pela técnica da réplica pode conter muitos defeitos decorrentes do processamento. A análise microestrutural revela filamentos ocos, originados durante a eliminação da esponja orgânica, além de microtricamento, porosidade e outros tipos de defeitos internos. Desse modo, há um consenso geral de que a melhoria das propriedades dos filamentos resulta em um melhor desempenho mecânico do filtro cerâmico [4-6]. O aumento da resistência mecânica, no entanto, deve ser obtido através do fortalecimento dos filamentos sem redução da permeabilidade do filtro. Sendo assim, a análise dos valores de resistência mecânica dos filtros não pode ser considerada isoladamente, uma vez que para filtros cerâmicos esta propriedade tem que estar associada a uma permeabilidade adequada.

Dentro deste contexto, foi avaliado neste trabalho o efeito das condições de processamento cerâmico na permeabilidade e resistência termomecânica dos filtros. Esta avaliação compreendeu a fabricação e caracterização de filtros cerâmicos de 8 ppi (poros por polegada linear) no sistema $\mathrm{Al}_{2} \mathrm{O}_{3}-\mathrm{SiC}$ produzidos a partir de suspensões cerâmicas com diferentes ajustes granulométricos. $\mathrm{O}$ ajuste granulométrico tem por objetivo otimizar o empacotamento 
das partículas nos filamentos sólidos e, consequentemente, melhorar as propriedades termomecânicas do filtro, porém, sem comprometer a permeabilidade do mesmo.

Neste estudo, a caracterização compreendeu tanto as propriedades reológicas das suspensões selecionadas, bem como a caracterização física, fluidodinâmica e mecânica dos filtros obtidos.

\section{MATERIAIS E MÉTODOS}

Inicialmente, selecionou-se quatro suspensões cerâmicas com distribuições granulométricas distintas. A suspensão de referência para o estudo comparativo foi baseada em uma composição comercial de filtros cerâmicos não ajustada granulometricamente, denominada Suspensão A, a qual apresentou a relação $\mathrm{Al}_{2} \mathrm{O}_{3}-\mathrm{SiC}$ de 1:1 em peso e o volume de sólidos em suspensão de $50 \%$. O ajuste granulométrico das suspensões B, C e D foi efetuado pelo programa PSDesigner, considerando-se o modelo de Andreasen com coeficiente de distribuição $\mathrm{q}_{\text {And }}=0,37$. O modelo de Andreasen assume que todos os tamanhos de partículas estão presentes em distribuições reais considerando a existência de partículas infinitesimais [7, 8]. Portanto, este é um modelo de abordagem contínua de distribuição granulométrica.

Para um melhor ajuste das suspensões à curva teórica de empacotamento, aumentou-se o teor de partículas finas nas suspensões B, C e D. A composição melhor ajustada apresentou, assim, o menor valor da diferença quadrática acumulada, uma vez que o modelo adotado considera a abordagem contínua de distribuição granulométrica. A Tabela I apresenta as composições das suspensões estudadas. $\mathrm{O}$ dispersante utilizado na defloculação das suspensões foi o polimetacrilato de sódio. Estas suspensões foram inicialmente caracterizadas quanto às suas propriedades reológicas em um viscosímetro Brookfield PV-III versão $3.1 \mathrm{LV}$ e, em seguida, utilizadas na produção dos filtros pelo método da réplica de esponjas [9].

Baseando-se em um trabalho anterior [10], no qual foi verificado que as propriedades fluidodinâmicas e mecânicas dos filtros são otimizadas quando a abertura da calandra está entre 20 e $30 \%$ da espessura da esponja, fixou-se neste estudo a abertura da calandra em 25\%. Após a impregnação e calandragem das esponjas poliméricas, estas foram secas a $100^{\circ} \mathrm{C}$ em estufa, calcinadas a 900 ${ }^{\circ} \mathrm{C}$ para eliminação dos aditivos orgânicos e, finalmente, os filtros foram sinterizados a $1250{ }^{\circ} \mathrm{C}$ por 5 horas ao ar. Após a produção dos filtros, estes foram caracterizados quanto às suas propriedades físicas, fluidodinâmicas e mecânicas e os resultados comparados ao do melhor filtro comercial.
Caracterização reológica. As medidas reológicas compreenderam as determinações da viscosidade aparente $(\eta)$ e da tensão de cisalhamento $(\sigma)$ em função da taxa de cisalhamento $(\dot{\gamma})$ para as suspensões da Tabela I. A partir das curvas de tensão $(\sigma)$ em função da taxa de cisalhamento $(\dot{\gamma})$, determinou-se os valores da tensão de escoamento $\left(\sigma_{\text {esc }}\right)$ pelo método de Casson. Segundo a literatura [11], o método de Casson é o mais indicado para avaliar o comportamento reológico de suspensões com alta concentração de sólidos, onde observa-se um comportamento não-linear da tensão em função da taxa de cisalhamento. A equação que rege o modelo de Casson é expressa como:

$$
(\sigma)^{1 / 2}=\left(\sigma_{\text {esc }}\right)^{1 / 2}+(\dot{\eta} \gamma)
$$

Caracterização física. A característica estrutural mais comum de um filtro é a sua densidade relativa, $\left(\rho / \rho_{\mathrm{s}}\right)$, onde (é a densidade geométrica do filtro e ( $\mathrm{s}$, a densidade teórica do material que o constitui. A fração de vazios no filtro determina sua porosidade, expressa por $\left[1-\left(\rho / \rho_{\mathrm{s}}\right)\right]$. A densidade geométrica foi calculada a partir das dimensões e massa dos corpos-de-prova e, a densidade teórica, foi determinada pela regra da mistura das matérias-primas.

Caracterização fluidodinâmica. Após a caracterização física, os filtros foram avaliados quanto à permeabilidade em um equipamento hidrodinâmico desenvolvido em laboratório. As constantes de permeabilidade $\left(\mathrm{k}_{1}\right.$ e $\left.\mathrm{k}_{2}\right)$ foram determinadas pela equação de Forchheimer para fluidos incompressíveis:

$$
\frac{\Delta \mathrm{P}}{\mathrm{L}}=\frac{\mu}{\mathrm{k}_{1}} \mathrm{v}_{\mathrm{s}}+\frac{\rho}{\mathrm{k}_{1}} \mathrm{v}_{\mathrm{s}}^{2}
$$

onde $\Delta \mathrm{P}$ é a queda pressão através do filtro; L é a espessura do filtro; $\mu$ e $\rho$ são, respectivamente, a viscosidade e a densidade do fluido; $\mathrm{v}_{\mathrm{s}}$, a velocidade superficial do fluido obtida pela razão entre a vazão volumétrica e a área da secção transversal ortogonal ao escoamento. As constantes $\mathrm{k}_{1}$ e $\mathrm{k}_{2}$ são chamadas de permeabilidade Darciana e não-Darciana, respectivamente. Os termos da equação (B) apresentam, nessa ordem, as contribuições viscosas e inerciais à queda de pressão total do fluido. A predominância de um ou outro termo na queda de pressão para um dado fluido é função da velocidade utilizada e das constantes $\mathrm{k}_{1}$ e $\mathrm{k}_{2}$.

\begin{tabular}{|c|c|c|c|c|}
\hline \multirow{2}{*}{ Matérias-Primas } & \multicolumn{3}{|c|}{ Composição (\% em peso) } & \multirow[b]{2}{*}{ Suspensão D } \\
\hline & Suspensão A & Suspensão B & Suspensão C & \\
\hline $\mathrm{SiC} 320 \mathrm{FE}^{\text {唃 }}$ & 46,00 & 46,50 & 46,52 & 48,74 \\
\hline $\mathrm{Al}_{2} \mathrm{O}_{3}$ 类(APC 3017G+A1000SG) & 46,00 & 46,40 & 46,37 & 44,12 \\
\hline 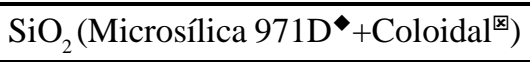 & 8,00 & 8,28 & 8,28 & 8,31 \\
\hline Aditivos Inorgânicos & 3,50 & 2,33 & 2,33 & 2,34 \\
\hline Aditivos Orgânicos & 0,05 & 0,24 & 0,24 & 0,24 \\
\hline $\mathrm{H}_{2} \mathrm{O}$ & 29,89 & 29,74 & 29,74 & 29,87 \\
\hline Diferença Acumulada Quadrática & 0,387 & 0,062 & 0,053 & 0,052 \\
\hline
\end{tabular}

Tabela I - Composições das suspensões estudadas. 
Caracterização mecânica. Compreendeu a avaliação da resistência mecânica sob compressão uniaxial dos filtros cerâmicos após sinterização. Os ensaios de compressão foram realizados em uma máquina universal de ensaios MTS 810, segundo a norma ASTM C133-94 com velocidade da travessa de $1,3 \mathrm{~mm} / \mathrm{min}$. As superfícies da amostra ortogonais à aplicação da carga de compressão foram cobertas com uma borracha rígida. Isso é recomendado para eliminar os efeitos de carregamento localizado, devido à topografia da superfície das amostras [6].

\section{RESULTADOS E DISCUSSÃO}

Inicialmente, fez-se uma análise das características reológicas das suspensões A, B, C e D. Posteriormente, esses resultados foram correlacionados aos de permeabilidade e resistência mecânica dos filtros produzidos.

Na Fig. 1 nota-se que todas as suspensões apresentam comportamento pseudoplástico, ou seja, a viscosidade aparente $(\eta)$ diminui à medida que a taxa de cisalhamento $(\dot{\gamma})$ aumenta. Observase também que a suspensão A apresenta viscosidade levemente inferior às suspensões $\mathrm{B}, \mathrm{C}$ e $\mathrm{D}$ em toda a faixa da taxa de cisalhamento analisada. Este comportamento deve ser decorrente do teor superior de partículas finas nas suspensões ajustadas granulometricamente.

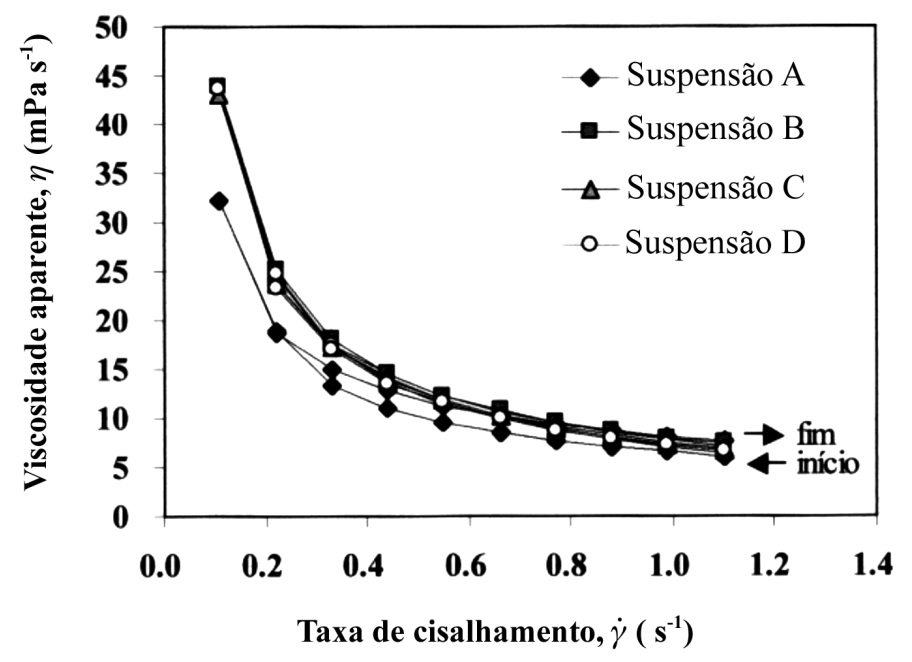

Figura 1: Curvas de viscosidade aparente $(\eta)$ versus taxa de cisalhamento $(\dot{\gamma})$ das suspensões A, B, C e D.

[Figure 1: Apparent viscosity ( $\eta$ ) as a function of shear rate $(\gamma)$ for suspensions A, $B, C$ and $D]$.

Ainda em relação a suspensão A, vê-se que suas curvas de viscosidade inicial e final (indicadas pelas setas na Fig. 1) em função da taxa de cisalhamento não são coincidentes, sendo os valores finais de viscosidade superiores àqueles da curva inicial. Este comportamento é típico de suspensões que têm suas propriedades reológicas dependentes do tempo, especificamente suspensões reopéxicas [12]. A reopexia é caracterizada pelo aumento da viscosidade aparente da suspensão em função do tempo. Tal fenômeno ocorre quando suspensões contendo aglomerados fracos, por exemplo, são inicialmente mantidas em um intenso cisalhamento por um longo intervalo e, em seguida, submetidas a baixas taxas em um período relativamente curto de tempo. Neste caso, uma parcela das partículas da suspensão, submetida a baixa taxa de cisalhamento, se une gradativamente para a formação de novos aglomerados, que aumentam a viscosidade aparente da suspensão em função do tempo. Acredita-se que a característica reopéxica da suspensão A é decorrente da presença de uma quantidade superior do aditivo inorgânico (3\%-p) em relação às demais composições (vide Tabela I), pois este aditivo atua como agente espessante em suspensões aquosas.

Na Fig. 2 é apresentado o comportamento da tensão de cisalhamento $(\sigma)$ em função da taxa de cisalhamento $(\dot{\gamma})$ para as suspensões avaliadas. A partir das curvas da Fig. 2, calculou-se a tensão de escoamento $\left(\sigma_{\text {esc }}\right)$ pelo método de Casson (equação A). Os valores obtidos são apresentados na Tabela II.

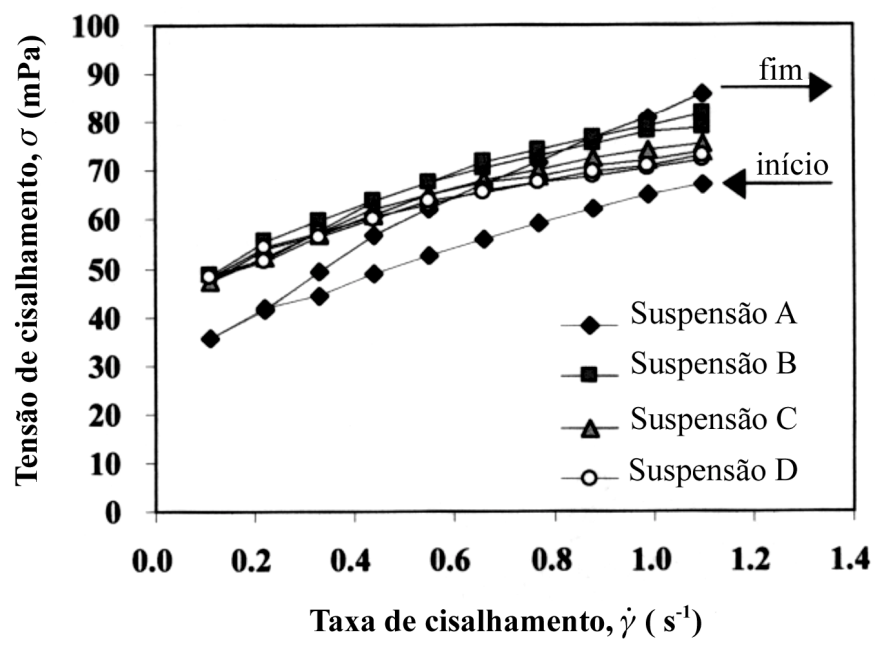

Figura 2: Comportamento da tensão $(\sigma)$ em função da taxa de cisalhamento $(\dot{\gamma})$ para as suspensões A, B, C e D.

[Figure 2: Shear stress $(\sigma)$ behavior as a function of shear rate $(\gamma)$ for suspensions $A, B, C$ and $D]$.

Tabela II - Valores de tensão de escoamento $\left(\sigma_{\text {esc }}\right)$ calculados para as suspensões $\mathrm{A}, \mathrm{B}, \mathrm{C}$ e $\mathrm{D}$ pelo método de Casson.

\begin{tabular}{lc}
\hline Suspensão & Tensão de escoamento, $\boldsymbol{\sigma}_{\text {esc }}(\mathbf{m P a})$ \\
$\mathrm{A}$ & 23,7 \\
\hline $\mathrm{B}$ & 36,6 \\
\hline $\mathrm{C}$ & 38,2 \\
\hline $\mathrm{D}$ & 39,5 \\
\hline
\end{tabular}

Segundo Casson [11], as partículas em uma suspensão com alto teor de sólidos tendem a formar aglomerados fracos devido à atração mútua entre as mesmas. Assim, para que a suspensão escoe é necessário aplicar uma tensão de intensidade suficiente para romper estes aglomerados. Deste modo, a tensão de escoamento pelo método de Casson pode ser considerada como um parâmetro que indica o grau de floculação da suspensão. Neste contexto, a suspensão A, não ajustada granulometricamente, apresentou o menor grau de floculação dentre todas as composições.

Ainda quanto à Fig. 2, observa-se que a curva da suspensão A apresenta uma histerese no comportamento da tensão em função da taxa de cisalhamento. Este comportamento para a suspensão A é típico de suspensão reopéxica. Como mencionado anteriormente, acredita-se que a reopexia da suspensão A é decorrente da presença 
do aditivo inorgânico espessante. Vê-se que a tensão medida na curva inicial é menor que a da curva final em toda a faixa da taxa de cisalhamento analisada. Isto porque na curva inicial a suspensão vem de um estado de alto cisalhamento e, portanto, bem dispersa. Já na curva final a suspensão permaneceu, por um período curto, sob baixo cisalhamento $\left(\sim 0,1 \mathrm{~s}^{-1}\right)$ o que possibilitou o rearranjo da rede de aglomerados. Assim, para romper esta rede de aglomerados e diminuir a tensão de cisalhamento atingindo o valor obtido na curva inicial, seria necessário deixar a suspensão por um período longo a uma taxa de cisalhamento alto. A confirmação de que a suspensão A tem suas propriedades reológicas dependentes com o tempo foi obtida medindo-se a tensão de escoamento das suspensões estudadas em função do tempo (Fig. 3), com intervalos de um minuto entre cada medida.

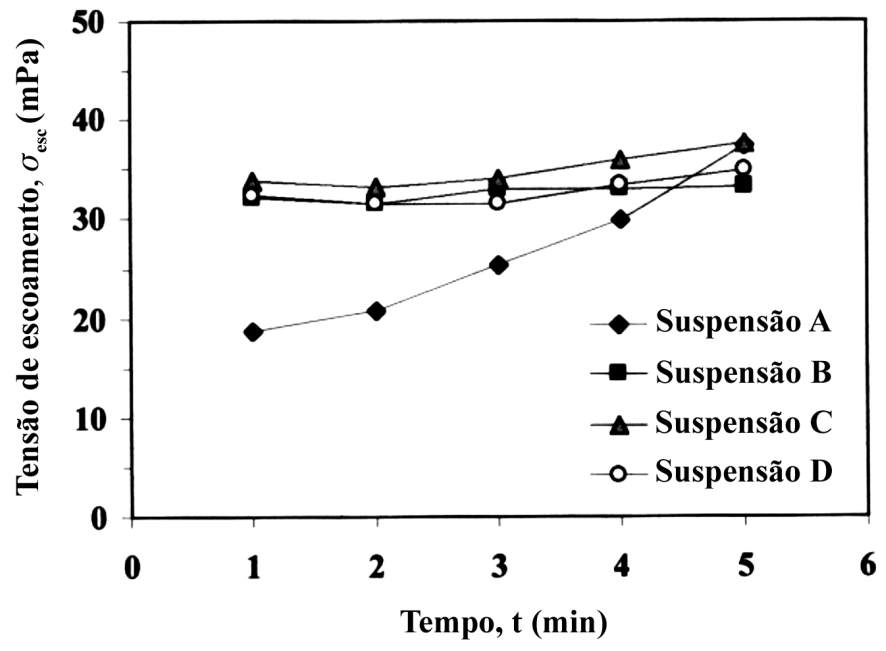

Figura 3: Comportamento da tensão de escoamento em função do tempo para as suspensões A, B, C e D.

[Figure 3: Yield stress behavior as a function of time for suspensions A, B, C and D].

Portanto, da análise das propriedades reológicas das suspensões estudadas, pode-se afirmar que, embora a suspensão A apresente propriedades $\left(\eta\right.$ e $\left.\sigma_{\text {esc }}\right)$ mais adequadas à fabricação dos filtros cerâmicos, é necessário um controle de suas características reológicas durante o processo de impregnação das esponjas, uma vez que tais características alteram-se com o tempo. Caso tal controle não seja feito, produzir-se-á filtros com propriedades físicas distintas, tais como densidade a verde e diâmetro de poro, e conseqüentemente com variações em suas propriedades após queima.

Conforme já mencionado, o objetivo deste trabalho foi a utilização do ajuste granulométrico como ferramenta para melhorar a resistência mecânica dos filtros, porém sem alterar a permeabilidade. No entanto, os valores médios das constantes de permeabilidade Darciana k (Fig. 4a) e não-Darciana k $_{2}$ (Fig. 4b) para os filtros analisados indicam que há diferenças na permeabilidade dos filtros cerâmicos. Os filtros produzidos com a suspensão A são mais permeáveis que os demais na faixa de escoamento viscoso $\left(\mathrm{k}_{1}\right)$, conforme ilustrado na Fig. 4a. Já quanto à constante de permeabilidade não-Darciana $\left(\mathrm{k}_{2}\right)$, observa-se na Fig. $4 \mathrm{~b}$ que os filtros das suspensões A e D apresentam uma boa permeabilidade no escoamento inercial, comparável aos valores obtidos para os filtros comerciais.
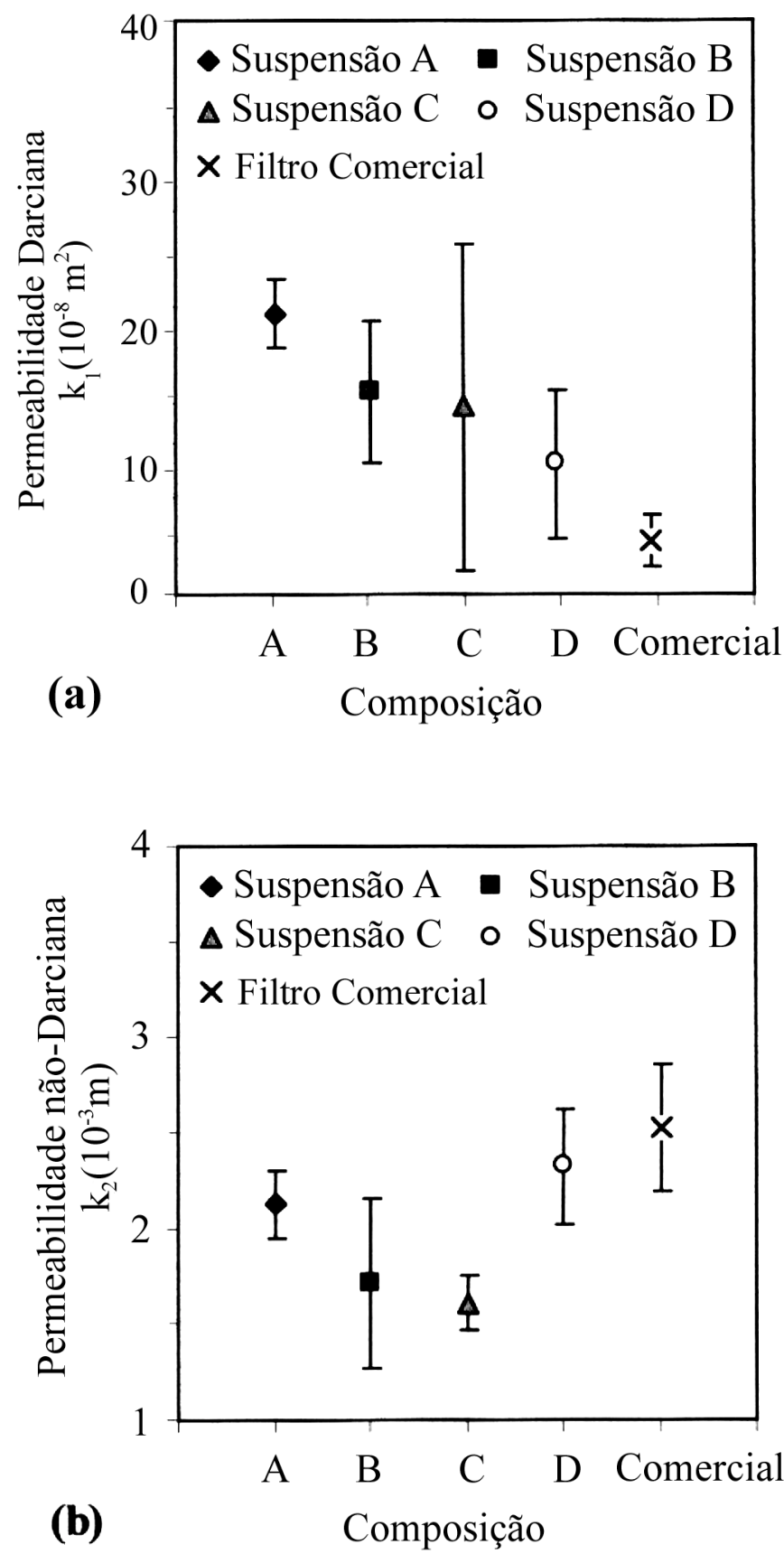

Figura 4: Valores médios da permeabilidade Darciana $\mathrm{k}_{1}$ (a) e não-Darciana $\mathrm{k}_{2}$ (b) obtida para os filtros das suspensões A, B, C, D e Filtro comercial em meio aquoso. [Figure 4: Average Darcian $k_{1}(a)$ and Non-Darcian $k_{2}$ (b) permeability constants in water flow for the commercial filter and for those obtained from suspensions $A$, $B, C, D]$.

Contudo, em processos de purificação de fluidos, os filtros cerâmicos devem apresentar não somente alta permeabilidade e eficiência de filtragem, mas também uma boa resistência mecânica. Estes parâmetros são influenciados diretamente pela estrutura celular. Segundo a literatura [4-6], o tamanho de poro (ou célula) não influencia a resistência mecânica dos filtros cerâmicos. Ao invés disso, é atribuído à "qualidade" dos filamentos dos filtros o desempenho dos mesmos sob solicitações mecânicas. O termo "qualidade" compreende a existência, ou não, de trincas, porosidade, da composição química e a microestrutura dos filamentos.

Neste contexto, faz-se a seguir uma discussão do desempenho 
mecânico dos filtros produzidos e, posteriormente, estes resultados serão avaliados em conjunto com os resultados de permeabilidade.

$\mathrm{Na}$ Fig. 5 é ilustrada a resistência mecânica média à compressão uniaxial $\left(\sigma_{\mathrm{fc}}\right)$ dos filtros produzidos a partir das suspensões $\mathrm{A}, \mathrm{B}$, $\mathrm{C}, \mathrm{D}$ e do filtro comercial, medida a temperatura ambiente. Os valores apresentados correspondem à média de cinco amostras para cada suspensão estudada. Nota-se que os filtros das suspensões A e $\mathrm{B}$ apresentam uma boa resistência mecânica média dentre o conjunto das amostras analisadas, inclusive superior aos valores obtidos para o filtro comercial.

Entretanto, baseando-se na teoria de empacotamento, esperavase que os filtros produzidos com as suspensões ajustadas granulometricamente $(\mathrm{B}, \mathrm{C}$ e $\mathrm{D})$ fossem os mais resistentes, uma vez que distribuições de tamanho de partículas que favorecem a obtenção de corpos densos apresentam menor retração volumétrica do corpo cerâmico durante a queima e melhor sinterabilidade, pois os poros na estrutura são menores podendo ser eliminados mais facilmente.

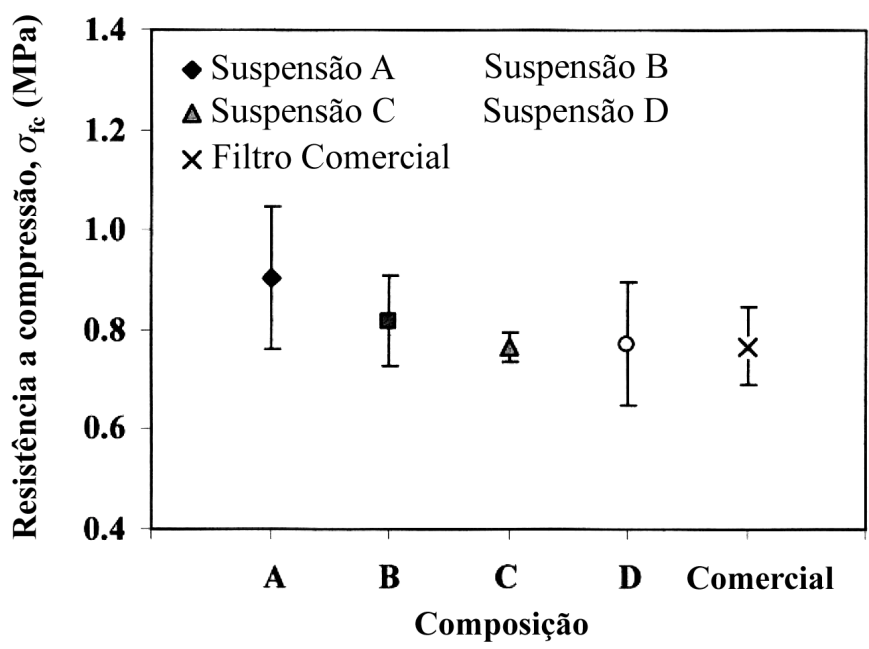

Figura 5: Resistência mecânica à compressão $(\sigma f)$ dos filtros produzidos a partir das suspensões A, B, C, D e Filtro comercial.

[Figure 5: Mechanical strength $\left(\sigma f_{c}\right)$ for the commercial filter and for those obtained from suspensions $A, B, C, D]$.

Assim, de acordo com os resultados da Fig. 5 pode-se fazer algumas suposições sobre o desempenho mecânico dos filtros:

1. O melhor empacotamento das partículas para as suspensões $\mathrm{B}, \mathrm{C}$ e D pode ter dificultado a eliminação da esponja polimérica durante a etapa de calcinação. Desse modo, a eliminação pode ter gerado um maior trincamento dos filamentos cerâmicos ainda não sinterizados.

2. $\mathrm{O}$ aumento no teor de finos nas suspensões ajustadas $(\mathrm{B}, \mathrm{C}$ e D) não garantiu por si só o aumento da resistência mecânica dos filtros, devendo ser também acompanhado por um aumento na quantidade do aditivo inorgânico (espessante), uma vez que este promove a sinterização do corpo cerâmico por fase vítrea [9, 13]. Porém, a quantidade ótima do espessante na composição deve ser determinada considerando-se, além das propriedades reológicas da suspensão, a resistência mecânica a quente dos corpos.

3. Já para a suspensão A, a superior quantidade do espessante inorgânico (vide Tabela I) proporcionou aos filtros uma melhor sinterabilidade dos filamentos e, conseqüentemente, um bom desempenho mecânico a frio. Contudo, acredita-se que o desempenho mecânico dos filtros desta composição possa ser ainda melhorado através do aumento da espessura dos filamentos cerâmicos. Segundo Brown et al. [14], filamentos mais espessos têm uma maior resistência ao trincamento gerado durante a eliminação da esponja polimérica. Assim, acredita-se que com o aumento da espessura ter-se-ía um recobrimento cerâmico ainda permeável à saída do polímero, uma vez que a composição A não é ajustada granulometricamente, porém de superior resistência mecânica.

Finalmente, com o intuito de avaliar as modificações simultâneas obtidas na permeabilidade e resistência mecânica dos filtros produzidos, calculou-se o parâmetro de otimização (P.O.), segundo estabelecido por Salvini et al. [10] para cada composição. O parâmetro de otimização (P.O.) é definido como:

$$
\mathrm{P} . \mathrm{O}=\frac{\Delta \mathrm{P}_{\min }}{\Delta \mathrm{P}_{\mathrm{i}}} \cdot \frac{\sigma_{6 \mathrm{i}}}{\sigma_{\text {tamax }}}
$$

onde $\Delta \mathrm{Pi}$ corresponde à queda de pressão total através da amostra i a uma dada velocidade de escoamento (vs); $\Delta \mathrm{P}_{\min }$ é a queda de pressão mínima no conjunto de amostras na mesma velocidade de escoamento de referência $\left(\mathrm{v}_{\mathrm{s}}\right) ; \sigma_{\text {fci }}$, a resistência mecânica a compressão uniaxial da amostra i, e $\sigma_{\text {fcmáx }}$ é o maior valor de resistência mecânica obtido no conjunto de amostras. Conforme indicado pela equação $(\mathrm{C})$, o parâmetro de otimização varia de 0 a 1. Quanto maior o valor deste parâmetro, melhor será a combinação entre permeabilidade e resistência mecânica para estas amostras.

Na Fig. 6 são ilustrados os valores calculados do parâmetro de otimização (P.O.) em função da velocidade de escoamento da água para os filtros das composições A, B, C e D e comercial.

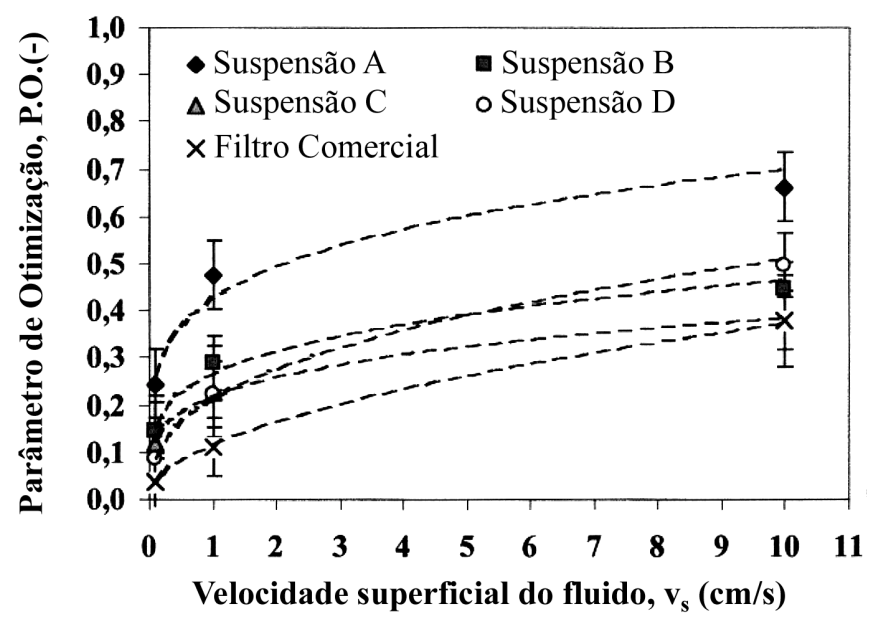

Figura 6: Parâmetro de otimização (P.O) para os filtros das suspensões A, B, C e D em função da velocidade de escoamento da água (vs).

[Figure 6: Optimization parameter (O.P.) evaluated as a function of water flow for filters obtained from suspensions $A, B, C$ and $D]$.

Observa-se na Fig. 6 que o parâmetro de otimização dos filtros produzidos com a suspensão A é maior dentre as demais em toda a faixa de velocidade de escoamento, inclusive superior ao dos filtros comerciais. Estes resultados para a suspensão A foram obtidos pela 
associação dos bons valores tanto de permeabilidade quanto de resistência mecânica. Nota-se também na Fig. 6 que, para uma mesma composição, o parâmetro de otimização (P.O.) aumenta com o aumento da velocidade de escoamento da água. Tal comportamento está associado à predominância do efeito inercial à medida que a velocidade de escoamento aumenta, no entanto não altera a classificação de desempenho dos filtros cerâmicos analisados.

De acordo com o objetivo inicial deste trabalho, aumentar a resistência mecânica sem comprometer a permeabilidade, pode-se concluir que a suspensão A apresentou o melhor conjunto de propriedades (permeabilidade e resistência mecânica) para a produção de filtros cerâmicos no sistema $\mathrm{Al}_{2} \mathrm{O}_{3}-\mathrm{SiC}$.

\section{CONCLUSÕES}

As principais conclusões deste trabalho são:

A distribuição granulométrica das suspensões cerâmicas é uma importante ferramenta na avaliação do desempenho dos filtros, uma vez que ela influencia tanto as características reológicas da suspensão cerâmica quanto as propriedades finais do produto.

A suspensão A, isenta de um cuidadoso ajuste granulométrico, apresentou valores de viscosidade e de tensão de escoamento mais adequados à fabricação dos filtros cerâmicos. Porém, é necessário um controle de suas características reológicas durante o processo de impregnação, uma vez que tais características alteram-se com o tempo.

A suspensão A apresentou o melhor conjunto de propriedades para a produção dos filtros cerâmicos no sistema $\mathrm{Al}_{2} \mathrm{O}_{3}-\mathrm{SiC}$, uma vez que os filtros obtidos desta suspensão apresentaram uma adequada permeabilidade associada a uma boa resistência mecânica. A otimização das propriedades para os filtros foi decorrente da maior facilidade de eliminação do polímero orgânico durante a calcinação.

\section{AGRADECIMENTOS}

Os autores deste trabalho agradecem a Alcoa Alumínio S.A. e a FAPESP pelo apoio dado à realização desta pesquisa.

\section{REFERÊNCIAS}

[1] M. D. M. Innocentini, Tese de Doutorado. UFSCar/ DEQ (1997). [2] L. J. Gibson, M. F. Ashby, Cambridge University Press. $2^{\text {nd }}$ Ed. (1997). [3] S. B. Bhaduri, Z. B. Qian, J. Mat. Synthesis and Processing 3, 6 (1995) 361-369.

[4] R. Brezny, D. J. Green, J. Am. Ceram. Soc. 74, 5(1991) 1061-1065.

[5] R. Brezny, D. J. Green, Acta Metall. 38, 12 (1990) 2517-2526.

[6] C. Q. Dam, R. Brezny, D. J. Green, J. Mater. Res. 5, 1 (1990) 163-171.

[7] F. S. Ortega, R. G. Pileggi, P. Sepulveda, V. C. Pandolfelli, Cerâmica 43, 283-284 (1997) 185-191.

[8] R. G. Pileggi, Dissertação de Mestrado. UFSCar/CCET/DEMa. (1996). [9] J. W. Brockmeyer, L. S. Aubrey, J. Dore, E. U. S. Pat. No. 4885263 (1989).

[10] V. R. Salvini, M. D. M. Innocentini, V.C. Pandolfelli, Am. Ceram. Soc. Bull. 79, 5 (2000) 49-54.

[11] L-C. Guo, Y. Zhang, N. Uchida, K. Uematsu, J. Am. Ceram. Soc. 81, 3 (1998) 549-556.

[12] I. R. Oliveira, A. R. Studart, R. G. Pillegi, V. C. Pandolfelli, "Dispersão e Empacotamento de Partículas - Princípios e Aplicações em processamento Cerâmico”. Ed. Fazendo Arte Editorial Primeira Edição (2000).

[13] J. Saggio-Woyansky, C. E. Scott, Am. Ceram. Soc. Bull. 71, 11 (1992) 1674-1682.

[14] D. D. Brown, D. J. Green, J. Am. Ceram. Soc. 77, 6 (1994) 1467-1472.

(Rec. 03/08/00, Ac. 10/11/00) 